\title{
Tunable Ultra Wideband Phase Shifter Using Liquid Crystal Polymer
}

\author{
Amin Abbosh ${ }^{1}$, Marek Bialkowski ${ }^{2}$, and David Thiel ${ }^{1}$ \\ ${ }^{1}$ Centre for Wireless Monitoring and Applications, Griffith University \\ Nathan QLD4111, Australia \\ aabbosh@ieee.org \\ ${ }^{2}$ School of ITEE, The University of Queensland \\ St Lucia QLD4072, Australia
}

\begin{abstract}
A tunable multilayer broadside-coupled phase shifter featuring ultra wideband (UWB) performance is presented. The tuning is accomplished using a DC biased Liquid Crystal Polymer (LCP) which is used for the construction of this device. Full electromagnetic-wave simulations show that the differential phase, referenced to a suitable length of a traditional microstrip line, can be varied between $40^{\circ}$ and $70^{\circ}$ across the band 3.1 to $10.6 \mathrm{GHz}$ by changing the biasing voltage across the LCP. The carried out simulations also show that the accomplished phase shift is accompanied by good quality insertion and return losses across the whole UWB.
\end{abstract}

Index Terms - Phase shifter, Liquid Crystal Polymer, Multilayer, broadside coupling.

\section{INTRODUCTION}

Phase shifters are important microwave devices that are used widely in electronic beam-scanning phased arrays, microwave instrumentation and measurement systems, and as modulators in communication sub-systems. Narrow and broadband type phase shifters can be accomplished using suitable design techniques.

In order to achieve the broadband operation of the phase shifters, the approach of coupled transmission lines is usually employed. One of the earliest designs that used the coupled lines method to construct broadband phase shifters is Schiffman differential phase shifter [1]. Other approaches to obtaining a broad bandwidth with an acceptable phase ripple include: cascaded multiple coupled sections [2] continuously tapered coupled section [3], a wiggling technique [4] and a multilayer broadside-coupled structure [5].

From the point of operation, phase shifters are divided into digital (binary) and analogue (continuously varied) types.With the rapid growth of digital techniques, the switched-type digital phase shifters in microwave integratedcircuit technology have been largely investigated [6]-[13]. The main design principle of this type of phase shifters is the use of a semiconductor switching device such as diode or transistor which toggles the microwave signal between various sections of microwave transmission lines. In [6], a switching network was combined with Schiffman phase shifter to build $180^{\circ}$ bit phase shifter. In [7], switching diodes were used to convert a microstrip line to a rectangular waveguide whereas in [8], a branch line coupler controlled by a varactor diode was used. Another example of the switched type phase shifter in the form of a three dimensional electromagnetic bandgap woodpile was reported in [9]. A recent alternative to diodes and transistors operating as switches are Micro Electro-Mechanical Systems (MEMS) [10]. They feature very low insertion losses up to the mmwave region.
In all switched types of phase shifters, insertion losses increase with the number of multiple switches that are required to obtain a desired phase shift range. In order to overcome this drawback, tunable (analogue type) phase shifters can be used. Varactors, ferrites and ferroelectrics [11] can be used as means of tuning. The advantage of varactors is that they offer a very fast tuning. However their disadvantage is that they handle only low power signals. Ferrites can deal with moderate power signals but require large currents and are slow in response, whereas ferroelectrics can provide a fast response; however, they require large voltages for tuning. The recently investigated Liquid Crystal Polymers (LCPs) show a similar property to ferroelectrics and exhibit a varying dielectric permittivity under varying biasing DC conditions but at a reduced voltage level [12]. This property offers the use of LCP to build various types of continuously tuned passive microwave device including phase shifters [13]. Although initially manufactured LCP featured a long switching time, the recent technological developments enable the manufacturing of LCP that have a very low switching time [14].

In this paper, a broadside-coupled multilayer configuration is used to design an ultra wideband phase shifter in LCP technology. The nematic LCP type BL006 is assumed as a DC tuned material. In the proposed device, it is used to fill the coupling gap between the top and bottom layers of the multi-layer structure of phase shifter. Full EM simulations of the designed device reveal its UWB characteristics.

\section{LCP PHASE SHIFTER}

The configuration of the proposed LCP phase shifter is shown in Fig. 1. The device uses a microstrip-slot technique to its construction, similar to the one described in [5]. It consists of two elliptical conducting patches, which are connected to the input and output microstrip lines, and facing each other at the top and bottom layers. The coupling between the patches is achieved via an elliptical slot in the ground plane which is located at the mid layer. The elliptical shape for the coupled structure is chosen because of its ability to achieve an almost constant coupling factor over the UWB [15]. The important modification presented in this paper is the inclusion of a liquid crystal polymer that fills the gap between the top and bottom layer. The added LCP is biased by applying a voltage to the two conducting plates. In order to isolate the biasing circuitry from RF, two RF chocks are used, as shown in Fig. 1. Also, to isolate the DC biasing from the input and output ports of the phase shifter, chip capacitors of $1 \mathrm{nF}$ are used as shown in Fig. 1. 


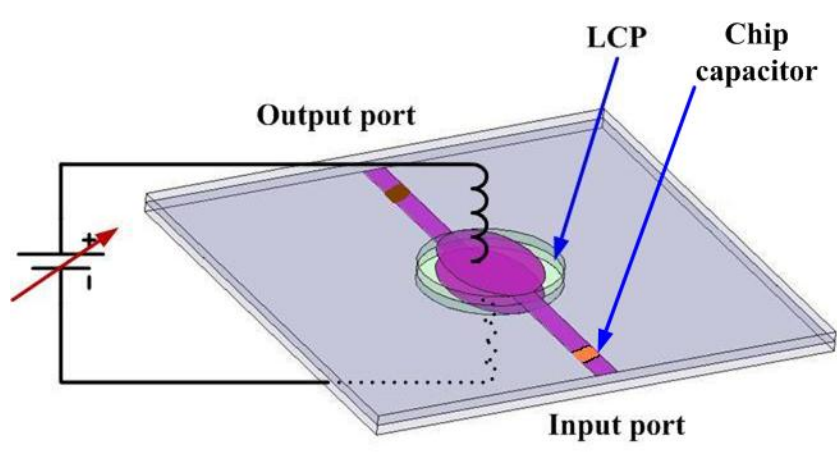

Fig.1 Configuration of the proposed phase shifter which employs the LCP as a tuning element.

According to the analysis presented in [5], the differential phase shift $(\Delta \Phi)$ with reference to a traditional microstrip line, which can be achieved using the proposed structure is;

$$
\Delta \Phi=90^{\circ}-2 \arctan \left[\frac{\sin \left(2 \pi \sqrt{\varepsilon_{e}} l / \lambda\right)}{\sqrt{1-C^{2}} \cos \left(2 \pi \sqrt{\varepsilon_{e}} l / \lambda\right)}\right]+\beta_{m} l_{m}
$$

where $C$ is the coupling factor, $l$ is the physical length of the coupled structure and $\mathcal{E}_{e}$ is the effective dielectric constant in the medium of the coupled structure. $l_{m}$ and $\beta_{m}$ are the physical length and phase constant of the reference microstrip line.

It is clear from (1) that the differential phase shift can be achieved by changing the effective dielectric constant of the material in the coupling medium. Following explanations in [5], this change is also responsible for variations in the coupling factor $C$. An increase of $\varepsilon_{e}$ is responsible for increasing the value of the second term in (1), which in turn leads to decreasing the differential phase shift.

Without a biasing voltage across the two terminals of the coupled structure, the LCP material has a dielectric constant $\varepsilon_{e \perp}$. This value depends on the type of LCP used. For the case of BL006, the nominal value is around 2.6 to 2.8 [12]. By increasing the biasing voltage across the LCP structure to around $50 \mathrm{~V}$ (this value depends on thickness of the utilized LCP layer), the molecules of the material start to align in the direction of the electrostatic field. This results in a different value for the effective dielectric constant, which in this case is called $\varepsilon_{e \uparrow}$. For BL006, the resulting dielectric constant can be in the range of 3.4 to 3.8 [12]. Continuous tuning between these two values of the dielectric constant, i.e. $\varepsilon_{e \perp}$ and $\varepsilon_{e \uparrow}$, is possible. It has to be noted that for frequencies above $400 \mathrm{MHz}$, the level of the applied microwave signal has no effect on this phenomenon. Thus, the only parameter that controls value of the effective dielectric constant of the coupling medium in the proposed structure, which operates across the range 3.1 to $10.6 \mathrm{GHz}$, is the DC biasing. This property of LCP offers the possibility of designing a continuously tuned phase shifter operating in the ultra wideband.

\section{RESULTS \& DISCUSSIONS}

In order to verify the above explained concept of tuned LCP phase shifter, the design method presented in [5] was applied. The dimensions of the phase shifter were chosen to achieve a $45^{\circ}$ assuming Rogers RO4003C (with $\varepsilon_{r}=3.38$, thickness $=0.508 \mathrm{~mm}$, and tangent loss $=0.0023$ ) as the substrate. In the next step, the liquid crystal polymer filling the gap between the top and bottom layer was assumed to be BL006 from MERCK [14]. Chip capacitors of $1 \mathrm{nF}$ were used to isolate the input and output ports from the DC biasing.

Variation of the differential phase shift across the ultra wideband frequency, as obtained from full EM simulations of the LCP phase shifter, is shown in Fig. 2. The results are for different values of the biasing voltage, and thus different values of the effective dielectric constant of the LCP.

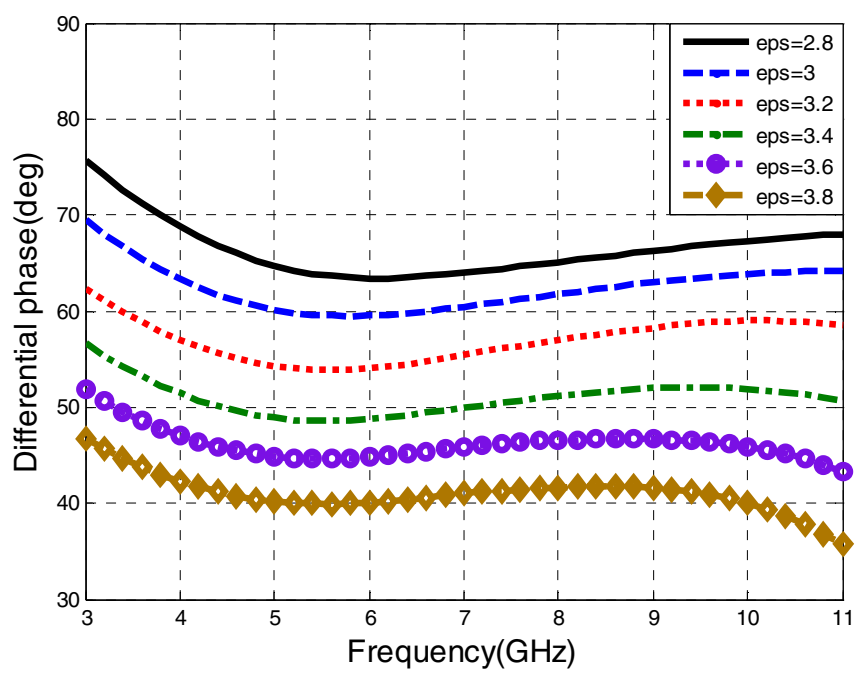

Fig.2 Variation of the differential phase shift with frequency for different biasing conditions (and thus different dielectric constants) of the LCP.

Fig.2 reveals that it is possible to design a phase shifter with wide tuning range of phase shift by just varying the DC biasing across the LCP. The achieved differential phase shifts have a fluctuation of only $\pm 3^{\circ}$ from the designated value across the band 3.1 to $10.6 \mathrm{GHz}$.

From the previous investigations into the characteristics of the LCP BL005, it has been shown that increasing the biasing voltage across the LCP, increases its dielectric constant [12]. Examining the design equations of a broadsidecoupled phase shifter [5] shows that increasing the dielectric constant of the material filling the gap between the top and bottom layer of the broadside-coupled structure increases the 
coupling factor which eventually decreases the differential phase shift as shown in (1). At the same time, increasing the dielectric constant of the filling medium (LCP in this case), increases the second term of (1) which also eventually decreases the differential phase shift. The simulated results of Fig. 2 confirm this conclusion where it is clear that increasing the dielectric constant decreases the achieved differential phase shift.

Designing a high performance phase shifter not only requires phase stability with the least deviation around the nominal value across the required bandwidth, but it also requires a low insertion loss and a high return loss across that band. Variation of the return loss and insertion loss across the UWB for different values of the LCP dielectric constant are shown in Figs. 3 and 4.

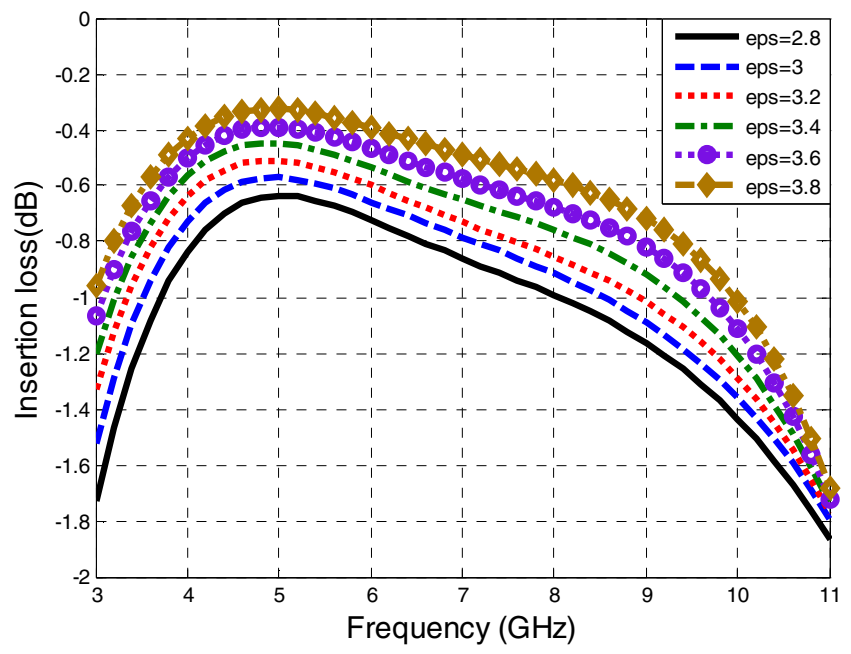

Fig.3 Variation of the insertion loss with frequency for different biasing conditions of the LCP.

As observed from the results presented in Fig.3, the insertion loss across the UWB is less than $0.4 \mathrm{~dB}$ at the centre of the UWB and it is less than $1 \mathrm{~dB}$ across the whole UWB when the maximum biasing voltage of $50 \mathrm{~V}$ DC is applied across the LCP to make its dielectric constant large. When the biasing voltage is zero, the dielectric constant of the LCP is around 2.8 and the insertion loss at the centre of the UWB is less than $0.8 \mathrm{~dB}$, while it is less than $1.7 \mathrm{~dB}$ across the whole UWB. The reason behind the decrease in the value of the insertion loss with increasing the biasing voltage comes from the fact that increasing the biasing voltage across the LCP to increase its dielectric constant results in a reduction in the loss tangent. It has been shown that for the LCP material used in this paper, i.e. BL006, the loss tangent is equal to 0.02 when the biasing voltage is adjusted to get a dielectric constant equal to 3.8 , while it is around 0.07 when the biasing voltage is low or zero to get a dielectric constant less than 3 [12].

The latest development in LC manufacturing shows that it is possible to produce materials with a loss tangent which is less than 0.01 [14]. If such a material is used in the proposed device, it is possible to show via simulations that the insertion loss across the whole UWB will be less than $0.5 \mathrm{~dB}$.

Concerning the return loss of the designed phase shifter, it is revealed from the results shown in Fig. 4 that the return loss is more than $15 \mathrm{~dB}$ at the centre of the UWB across the whole tuning range of the LCP. The return loss is less than 10 $\mathrm{dB}$ across the whole UWB and for different biasing conditions of the LCP.

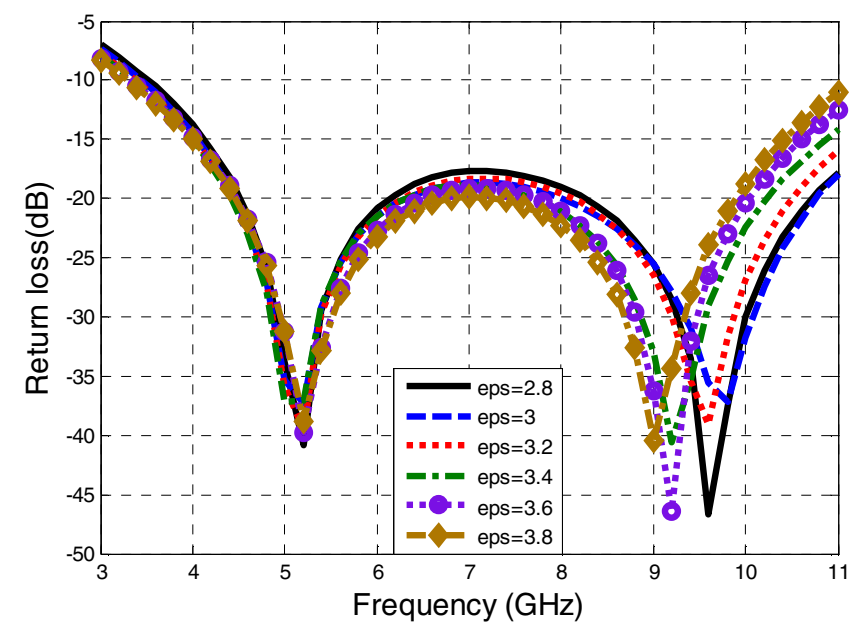

Fig.4 Variation of the return loss with frequency for different biasing conditions of the LCP.

The phase shifter presented in this paper is suggested for the applications that require a variable differential phase shift. However, it can also be useful for the applications which need a fixed differential phase shift. As noted from the results of Fig. 2, the differential phase shift is sensitive to even a small variation in the dielectric constant of the utilized substrate. Inspecting the datasheets of different types of substrates manufactured by various companies shows that there is always a possibility of at least $\pm 5 \%$ variation in the dielectric constant of the substrate compared with the typical value shown in the datasheet. This difference in the value between the typical and real values of the dielectric constant causes a significant difference between the designed and obtained phase shift as proven from Fig. 2. Thus, it is important even for the fixed phase shifter to have a tuning facility that can compensate for the variation in the dielectric constant and make the differential phase shift equal to the designed value.

\section{CONCLUSION}

A tunable phase shifter with ultra wideband performance in microstrip-slot technique has been presented. The device uses the broadside-coupled multilayer configuration to achieve the required performance. To tune the phase shifter, a liquid crystal polymer (LCP) is used to fill the gap between the top and bottom microstrip coupled layers. The dielectric properties of the LCP are varied by changing the biasing 
voltage across two conductive layers supporting the LCP. The simulated results presented in this paper have shown the possibility of achieving a high stable phase shift across the band 3.1 to $10.6 \mathrm{GHz}$ by just changing the DC biasing of the LCP. The device has an insertion loss which is less than $1 \mathrm{~dB}$, while the return loss is more than $15 \mathrm{~dB}$ across most of the whole ultra wideband.

\section{REFERENCES}

[1] B. Schiffman, "A new class of broad-band microwave 90-degree phase shifters," IRE Trans. Microwave Theory Tech., vol.6, no.4, pp. 232-237, 1958.

[2] B. Schiek, and J. Kohler, "A method for broad-band matching of microstrip differential phase shifters," IEEE Trans. Microwave Theory Tech., vol.25, no.8, pp.666$671,1977$.

[3] C. Tresselt, "Broad-band tapered-line phase shift networks," IEEE Trans. Microwave Theory Tech., vol. 16, no.1, pp. 51-52, 1968.

[4] J. Taylor, and D. Prigel," Wiggly phase shifters and directional couplers for radio-frequency hybridmicrocircuit applications," IEEE Trans. On Parts, Hybrids and Packaging, vol.12, no. 4, pp. 317-323, 1976.

[5] A. Abbosh, "Ultra-wideband phase shifters," IEEE Trans. Microwave Theory Tech., vol.55, no.9, pp.19351941, 2007.

[6] S. Eom, "Broadband $180^{\circ}$ bit phase shifter using $/ 2$ coupled line and parallel /8 stubs," IEEE Microwave and wireless components letters, vol.14, no.5, pp.228230, 2004.
[7] Z. Jin, S. Ortiz, and A. Mortazawi," Design and performance of a new digital phase shifter at X-band," IEEE Microwave and wireless components letters, vol.14, no.9, pp.428-430, 2004.

[8] S. Cheng, E. Öjefors, P. Hallbjörner, and A. Rydberg, "Compact reflective microstrip phase shifter for traveling wave antenna applications," IEEE Microwave and wireless components letters, vol.16, no.7, pp.413-433, 2006.

[9] A. Weily, T. Bird, K. Esselle and B. Sanders," Woodpile EBG phase shifter," Electronics Letters, vol.42, no.25, 2006.

[10]N. Barker, and G. Rebeiz, "Optimization of distributed MEMS transmission-line phase shifters-U-band and Wband designs," IEEE Trans. Microwave Theory Tech., vol. 48, pp. 1957- 1966, 2000.

[11]B. Acikel, et al., "A new high performance phase shifter using BaxSr1-TiO3 thin films," IEEE Microwave and wireless components letters, vol. 12, pp. 237-239, 2002.

[12] Y. Utsumi, and T. Kamei, "Dielectric permittivity measurements of liquid crystal in the microwave and millimeter wave ranges," Molecular Crystals and Liquid Crystals, vol. 409, no.1, pp.355 - 370, 2004.

[13] S. Mueller, et al., "Passive tunable liquid crystal finline phase shifter for millimeterwaves," Proc. 35th European Microwave Conf., pp. 297-300, 2005.

[14] Merck KGaA, Darmstadt, Germany, http://www.merckchemicals.com/ .

[15]A. Abbosh, and M. Bialkowski, "Design of compact directional couplers for UWB applications," IEEE Trans. Microwave Theory Tech., vol.55, no.2, pp.189-194, 2007.

[16]F. Goelden, et al., "Liquid-crystal varactors with fast switching times for microwave applications," Electronics letters, vol.44, no.7, 2008. 